Military Technical College

Kobry El-Kobbah,

Cairo, Egypt

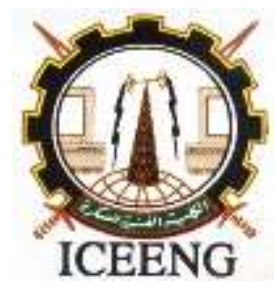

$11^{\text {th }}$ International Conference

on Electrical Engineering

ICEENG 2018

\title{
Behavior of Different Physiological Markers in Relation to Computer Based Mental Activities
}

\author{
Ayman A. Nassar ${ }^{\star}$, E. Elsamahy ${ }^{\star}$, A. Awadallah*, M. H. Elmahlawy**
}

\begin{abstract}
Mental stress is the response of human body subjected to any mental activities. Jobs, study, and major life changes can be classified as mental stressors, humans can be normally subjected to mental stress. However, paying attention and monitoring minor and major mental stress activities assist in protection from mental health risk and control human's performance.

Many researchers studied different responses from mental activities to identify the most related marker in relation to the subjected mental activity. However, it is still a challenge. One of the most frequently used computer based mental tasks is Stroop color test that shows a great influence on subjects' responses. In this paper, experimental comparison is applied between different responses of physiological markers in relation to laboratory mental stressor based on Stroop color test.
\end{abstract}

\section{KEY WORDS}

Mental Stress, Photo-Plethysmography, Pupil Diameter, Respiration, Stroop Color.

\section{Introduction}

There are many laboratory mental stressors used for stimulating autonomic nervous system (ANS) which can be used to create the suitable mental stress, such as visual search tasks, Stroop color test, games, automated Cabin Air Management System (aCAMS), arithmetic test, university exams, Air Traffic Control (ATC), and other mental tasks [1-7].

* Egyptian Armed Forces.

** Electrical Eng. Dep., Faculty of Engineering and Technology, Future University in Egypt. 
Stroop color test is one of the frequently used mental stressors in literature, J. Stroop designed it in 1935[8], and J. Stroop did this test by using colored cards. However, now it becomes a computer related test, this test based on identification of colors in trials, each trial has a certain time duration that adapted as required.

Mental stress produced due to Human Machine interaction had been studied by many researchers, aiming to establish a relationship between mental stress and different physiological signals of the subject. The physiological parameters that are frequently used, such as Electroencephalogram (EEG), Electrocardiogram (ECG), Galvanic Skin Response (GSR), Heart Rate (HR), Photo-plethysmography (PPG), and Respiration Rate (RR) [9-15]. Suitable markers are extracted from those parameters that show a response of the subjected mental stress.

This paper is organized as follows: Section II, discusses the material and used methods, while Section. Section III, shows the experiments' results, while Section IV manifest the conclusions and future work.

\section{Material and methods:}

\section{A. Experimental setup:}

Thirty-four healthy subject participated in the data acquisition experiments. All subjects are engineering students (post and undergrad students), with age of (29.8 \pm 6.7) years. The selection criteria of subjects stated that all selected subjects have no complaint about any critical diseases, right handed, pass colorblind test, and having experience in using computer for more than five years. Furthermore, exclusion criterion was applied to exclude subjects underwent any surgical operation in last 2 years, and subjects that have heart or blood pressure diseases.

All subjects signed consent forms before the start of the experiment. Moreover, some general conditions were applied to all subjects, such as subjects have to carry out the test before $12 \mathrm{PM}$, all the subjects were asked to sit on chair in front of a laptop computer with screen 15.4 inch, and the surrounding light intensity and direction was the same. Moreover, all subjects have to drink fruit juice and take rest for 3 minutes as a mandatory relaxation period before the experiment.

The experiment was carried out to identify the markers that are influenced by mental stress. MATLAB ${ }^{\circledR}$ was used as a software-programming tool to build a graphical user interface (GUI) that implement a Stroop color test and the analysis program. BIOPAC MP $1500^{\circledR}$ research system is used to acquire Photo-Plethysmography signal with module No. PPG100C, and Respiation signal with module No. RSP100C. Photo-Plethysmography is acquired with a sampling rate of $100 \mathrm{~Hz}$. Furthermore, The BIOPAC Hardware API tools allow MATLAB the capability of controlling BIOPAC research system. Moreover, Gazepoint ${ }^{\circledR}$ Model No. (GP3 Desktop) is used to detect subjects' pupil diameter with sampling frequency $61 \mathrm{~Hz}$. Fig. 1 shows the block diagram of the experimental setup including data flow paths and the acquisition devices used in the experiment.

Fig. 2 shows the flowchart of the experimental setup, the mandatory resting period is the period where the subject drinks fruit juice and this period is 3 minutes, the 
second period is the recorded resting period that takes 2.5 minutes and the subject watches a video based on seawater sound and natural sightseeing. However, the subject's physiological signals is recorded during his watching. The last period is the computer based mental stressor, at which the subject starts to answer the Stroop color test with recording his physiological signals.

B. Computer Based Mental Activities:

Stroop color test is selected as it is classified as a mental stressor with an adequate response and it needs very short time of training. The computer based mental stressor was performed as a word appears in front of the subject every trial with different color, the trial period is 1.2 second and the whole test takes 150 second. The answer of subjects is counted to a certain aspects, which are:

1. The trial is counted as correct if the subject correctly selected the correct button.

2. The trial is counted as wrong if the subject selected wrong button or did not select any button before the available trial time ends up.

C. Markers Extraction

Photo-Plethysmography signal underwent hardware filtration by using Biopac Research System with band pass filter between $0.5 \mathrm{~Hz}$ and $3 \mathrm{~Hz}$. Four markers were extracted from this signal which are Heart Rate (HR), Heart Rate Standard deviation (HRstd), Relative SpO2 value ( $\mathrm{SpO}_{2}$ ) which is Blood Volume Pulse (BVP) waveform via optical method, and R-R interval (R-Rint) which is the time between two successive $\mathrm{SpO}_{2}$ peaks.

Respiration signal is extracted by measuring the thoracic expansion and contraction while breathing and the signal underwent hardware filtration by using Biopac Research System with band pass filter between $0.5 \mathrm{~Hz}$ and $1 \mathrm{~Hz}$. Four markers were extracted from this signal which are Respiration Rate (RR), Respiration Rate Standard deviation (RRstd), Relative Respiration peak preassure value (ResP) which is peak values of pressure measured due to the expansion of the thoracic cage, and relative tidal volume ( $T V r)$ which is the corresponding value between the thoracic cage contraction and thoracic cage expansion.

Furthermore, two markers were extracted from Gazepoint ${ }^{\circledR}$, which are pupil diameter of the left eye (LPD), and pupil diameter of the right eye (RPD). Moreover, the recorded resting period is classified as the zero level for the proposed system. Consequently, zero level were subtracted from all the markers' values to monitor only the changes of the markers not the absolute values.

Fig. 3 shows the subject's results from Stroop color test and the captured physiological signals. Furthermore, signal processing and analysis were done to extract the markers that were used in this study. 


\section{Results and Discussion}

Analysis of Variance (ANOVA) is a statistical tool used to test differences between two or more samples' means, this tool monitor the inferences about means by analyzing variance.

In case of different subjects underwent the stimulated mental stress, the mental stress is called between-subjects mental stress. The term "between subjects" reflects the fact that comparisons are between different groups of subjects. However, in this study, the comparisons were not applied between different groups of subjects but applied between the same subjects. Consequently, when the same subjects underwent the stimulated mental stress, the mental stress is called within-subjects mental stress, or repeated-measure mental stress of the same subjects[16].

In this study, the only independent factor is the mental stress. Consequently, oneway ANOVA is used. If the null hypothesis is approved by ANOVA, it means that the subjects' means of specific marker are the same for resting period and when subjected to mental stress. However, if the null hypothesis is rejected, then it can be concluded that the subjects' means is different.

Table 1 shows the p-values of different physiological markers, the markers that approved the null hypothesis are HRstd, R-Rint, RRstd, ResP, and TVr. In other words, these markers didn't show any significant difference during mental stress $(P>0.05)$, other markers rejects the null hypothesis and show a slight significant difference $(P<0.05)$ such as $H R$, and $R R$. However, other markers such as $\mathrm{SpO}_{2}$, RPD, and LPD shows a great significant difference $(P<0.01)$ and shows the great influence of the mental stress on subjects' markers.

Furthermore processing were done to verify the significant with Tukey's Honestly Significant Difference test (HSD) in MATLAB ${ }^{\circledR}$ toolbox.

Fig.4 shows a graphical statistical comparing between subject's resting state and under influence of mental stress for different physiological markers, the disjoint intervals exhibits that there is a significant difference between the markers.

\section{Conclusion}

Detection and evaluation of mental stress is one of the challenges that faced researchers nowadays, the proposed work shows the response of different physiological markers. Pupil diameter and $\mathrm{Spo}_{2} \mathrm{r}$ value shows high significant change in response to mental stress and can be used as a mental stress detector, HRstd, RRint, RRstd, ResP, and TVr can not be classified as mental stress detector as it shows a poor response to mental stress. In the future, the response of the markers that shows great impact should be studied for different levels of mental stress. Consequently, building a model that can be used to quantify and evaluate mental stress. 


\section{Figures}

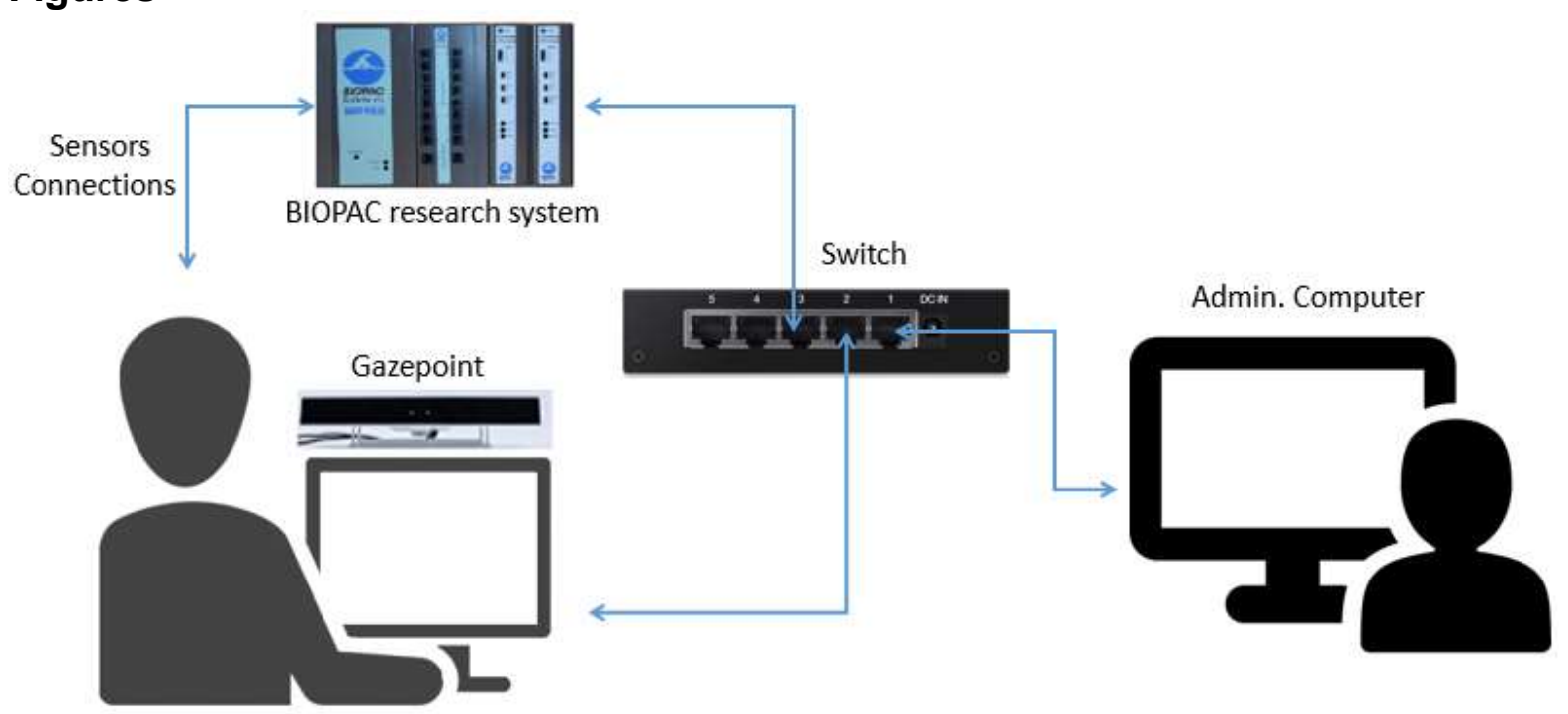

Subject's computer

Figure 1. Block diagram of the experimental setup and data flow.
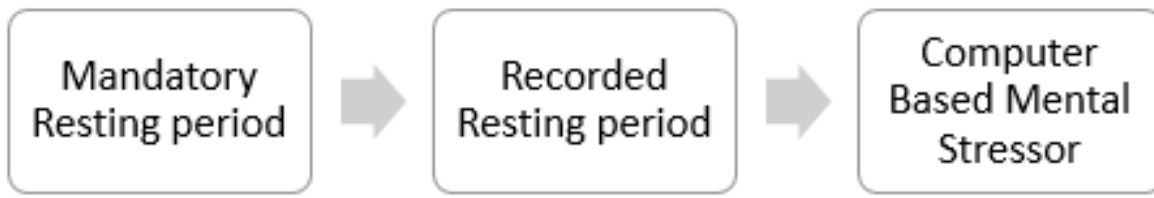

Figure 2. The experiment flowchart.

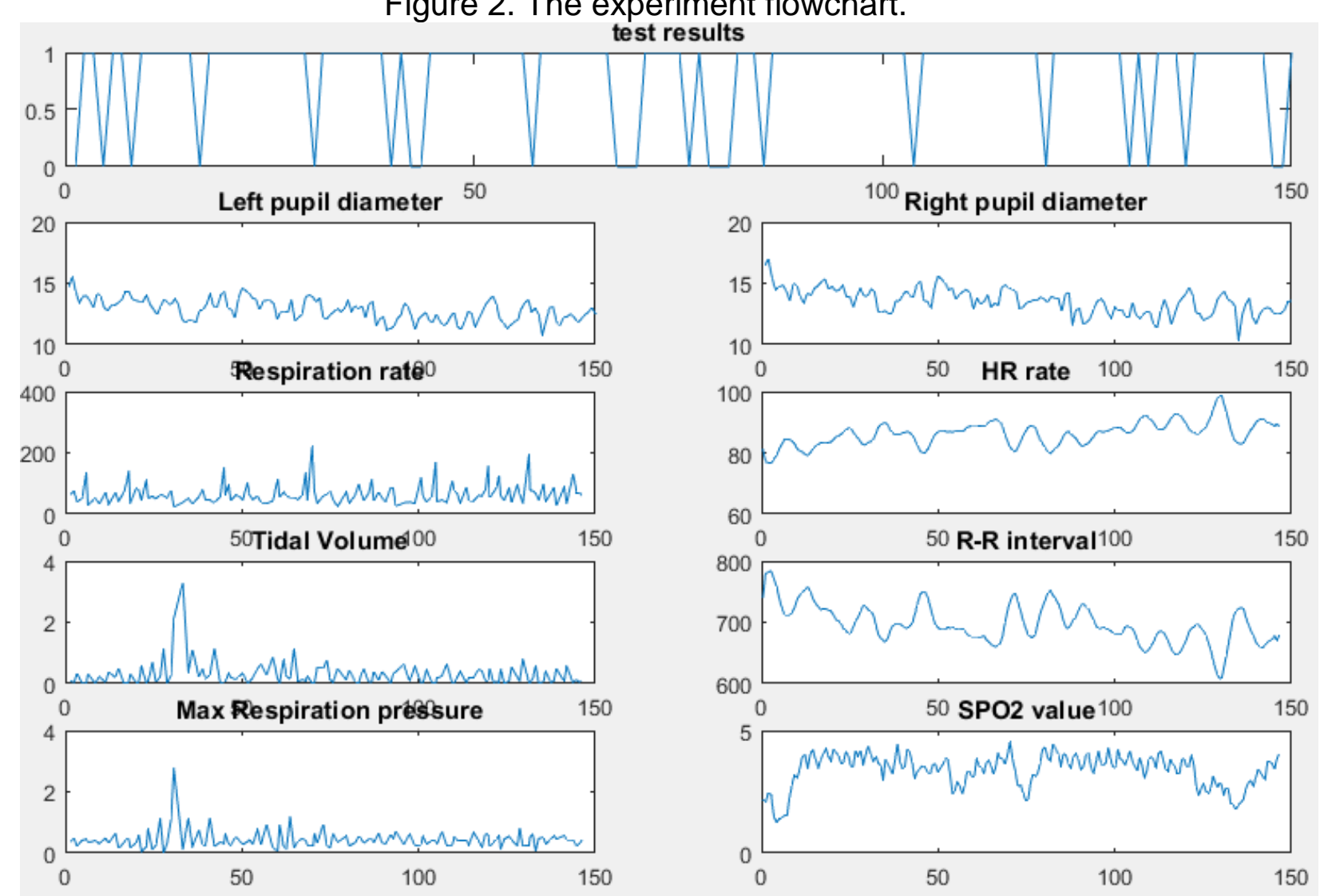

Figure 3. The GUI of the data analysis software. 

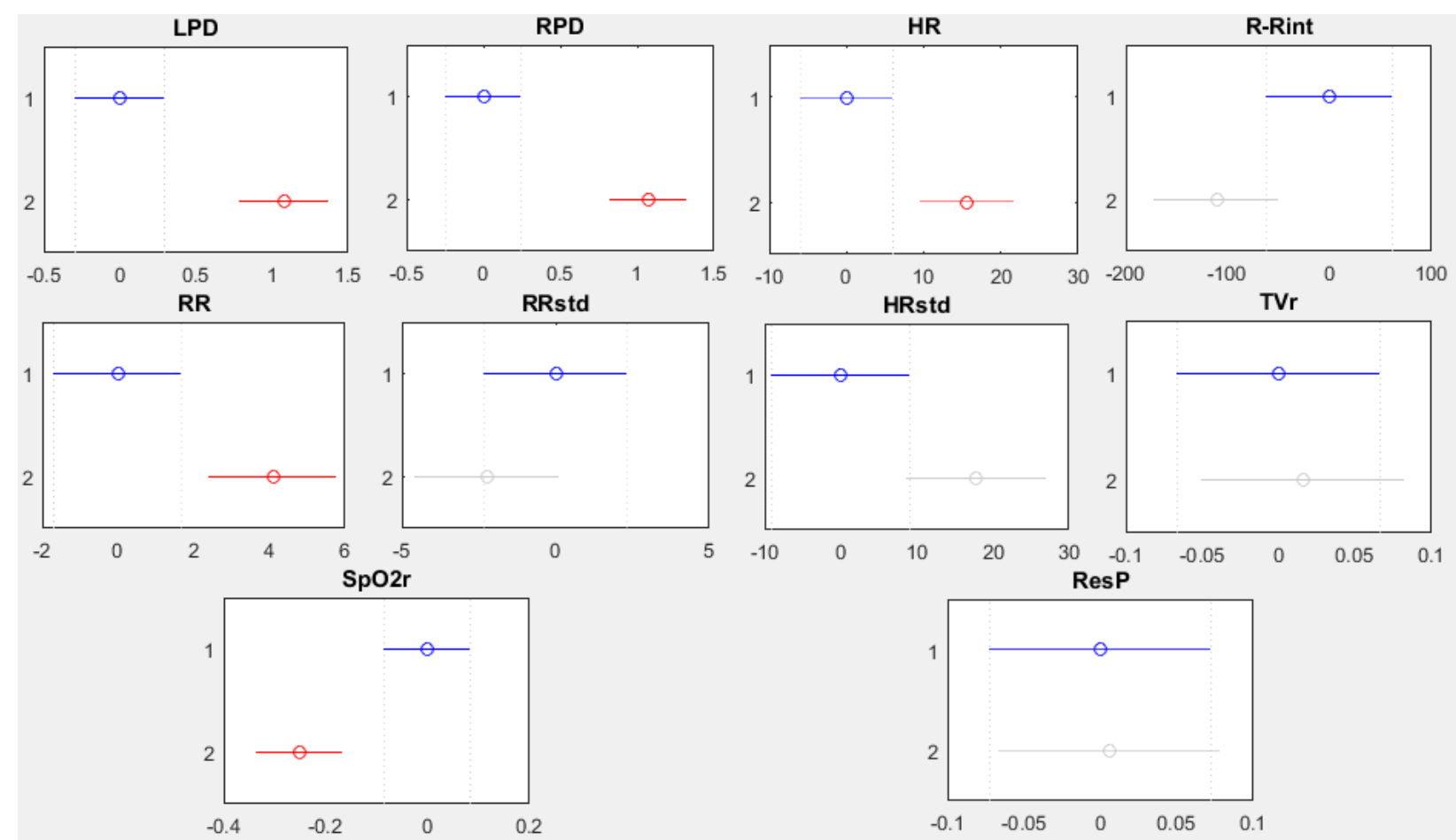

Figure 4. The comparison between subject's rest and under stress for different markers. 


\section{Tables}

Table 1. The p-values of different physiological markes.

\begin{tabular}{|c|c|c|c|c|}
\hline No. & Physiological signal & Marker & P-value & Conclusion \\
\hline 1 & \multirow{4}{*}{$\begin{array}{l}\text { Photo- } \\
\text { Plethysmography }\end{array}$} & $\mathrm{HR}$ & 0.0121 & $P<0.05$ \\
\hline 2 & & HRstd & 0.0551 & $p>0.05$ \\
\hline 3 & & $\mathrm{SpO}_{2} \mathrm{r}$ & 0.004 & $P<0.01$ \\
\hline 4 & & R-Rint & 0.0765 & $p>0.05$ \\
\hline 5 & \multirow{4}{*}{ Respiration } & RR & 0.0185 & $P<0.05$ \\
\hline 6 & & RRstd & 0.3469 & $p>0.05$ \\
\hline 7 & & ResP & 0.9348 & $p>0.05$ \\
\hline 8 & & $\mathrm{TVr}$ & 0.8121 & $p>0.05$ \\
\hline 9 & \multirow{2}{*}{ Pupil Diameter } & RPD & $5.5^{*} 10^{-5}$ & $\mathrm{P}<0.01$ \\
\hline 10 & & LPD & 0.0004 & $P<0.01$ \\
\hline
\end{tabular}




\section{References}

1. Castaldo, R., P. Melillo, and L. Pecchia. Acute Mental Stress Detection via Ultra-short term HRV Analysis. in World Congress on Medical Physics and Biomedical Engineering, June 7-12, 2015, Toronto, Canada. 2015. Springer.

2. Seoane, F., et al., Wearable biomedical measurement systems for assessment of mental stress of combatants in real time. Sensors, 2014. 14(4): p. 7120-7141.

3. Kumar, M., et al., Fuzzy evaluation of heart rate signals for mental stress assessment. IEEE transactions on fuzzy systems, 2007. 15(5): p. 791-808.

4. Ting, C.-H., et al., Real-time adaptive automation system based on identification of operator functional state in simulated process control operations. IEEE Transactions on Systems, Man, and Cybernetics-Part A: Systems and Humans, 2010. 40(2): p. 251-262.

5. El-Samahy, E., et al. A new computer control system for mental stress management using fuzzy logic. in Evolving and Adaptive Intelligent Systems (EAIS), 2015 IEEE International Conference on. 2015. IEEE.

6. Visnovcova, Z., et al., Complexity and time asymmetry of heart rate variability are altered in acute mental stress. Physiological measurement, 2014. 35(7): p. 1319.

7. Kobayashi, N., et al. A new mental stress detection method using biosignals under visual search tasks. in [Engineering in Medicine and Biology, 1999. 21st Annual Conference and the 1999 Annual Fall Meetring of the Biomedical Engineering Society] BMES/EMBS Conference, 1999. Proceedings of the First Joint. 1999. IEEE.

8. Stroop, J.R., Studies of interference in serial verbal reactions. Journal of experimental psychology, 1935. 18(6): p. 643.

9. $\mathrm{Xu}, \mathrm{X} .$, Analysis on Mental Stress/Workload Using Heart Rate Variability and Galvanic Skin Response during Design Process. 2014, Concordia University.

10. Lee, J.-H., Photoplethysmography Based Mental Stress Analysis Algorithm and System for Human Wellbeing Life. Sensors \& Transducers, 2017. 208(1): p. 28.

11. Gandhi, S., M.S. Baghini, and S. Mukherji. Mental stress assessment-a comparison between HRV based and respiration based techniques. in Computing in Cardiology Conference (CinC), 2015. 2015. IEEE.

12. Koyas, E., et al. Detection of intention level in response to task difficulty from EEG signals. in Machine Learning for Signal Processing (MLSP), 2013 IEEE International Workshop on. 2013. IEEE.

13. Saidatul, A., et al. Analysis of EEG signals during relaxation and mental stress condition using AR modeling techniques. in Control System, Computing and Engineering (ICCSCE), 2011 IEEE International Conference on. 2011. IEEE.

14. Boonnithi, S. and S. Phongsuphap. Comparison of heart rate variability measures for mental stress detection. in Computing in Cardiology, 2011. 2011. IEEE.

15. Sun, F.-T., et al. Activity-aware mental stress detection using physiological sensors. in International Conference on Mobile Computing, Applications, and Services. 2010. Springer.

16. Lane, M., et al., e-Statistics education: A multimedia course of study. Online Edition. Retrieved on, 2009. 18(1): p. 2014. 